\title{
Minimal positive realizations of transfer functions with nonnegative multiple poles
}

\author{
Béla Nagy Máté Matolcsi
}

Béla Nagy is Professor at the Mathematics Department of Technical University, Budapest, e-mail: bnagy@math.bme.hu. His work was supported by OTKA-T047276.

Máté Matolcsi is Research Assistant at the Alfréd Rényi Institute of Mathematics, Budapest, e-mail matomate@renyi.hu. His work was supported by OTKA-T047276, F049457, T049301. The present paper was supported by the Hungarian-Spanish Scientific and Technological Governmental Cooperation, with the support of the Research and Technological Development Fund of Hungary and the Ministry of Education of Spain. 


\begin{abstract}
This paper concerns a particular case of the minimality problem in positive system theory. A standard result in linear system theory states that any $n$ th-order rational transfer function of a discrete timeinvariant linear SISO system admits a realization of order $n$. In some applications, however, one is restricted to realizations with nonnegative entries (i.e. a positive system), and it is known that this restriction may force the order $N$ of realizations to be strictly larger than $n$. A general solution to the minimality problem (i.e. determining the smallest possible value of $N$ ) is not known. In this brief we consider the case of transfer functions with nonnegative multiple poles, and give sufficient conditions for the existence of positive realizations of order $N=n$. With the help of our results we also give an improvement of an existing result in positive system theory.
\end{abstract}

\title{
Keywords
}

Positive linear systems, discrete time filtering, minimal realizations

\section{INTRODUCTION}

Assume we are given the transfer function

$$
H(z)=\frac{p_{1} z^{n-1}+\ldots+p_{n}}{z^{n}+q_{1} z^{n-1}+\ldots+q_{n}} ; p_{j}, q_{j} \in \mathbb{R} \text { for all } 1 \leq \mathrm{j} \leq \mathrm{n}
$$

of a discrete time-invariant linear SISO system of McMillan degree $n$.

It is a standard result in linear system theory (see e.g. [10], Chapter 9) that an $n$th order realization of $H(z)$ (i.e. a triple $\mathbf{A} \in \mathbb{R}^{n \times n}$, b, c $\in \mathbb{R}^{n}$ such that $H(z)=\mathbf{c}^{T}(z \mathbf{I}-\mathbf{A})^{-1} \mathbf{b}$ holds) can always be constructed. In this note, however, we are interested in the positive realization problem, i.e. finding $\mathbf{A}, \mathbf{b}, \mathbf{c}$ with nonnegative entries. The nonnegativity restriction on the entries of $\mathbf{A}, \mathbf{b}, \mathbf{c}$ reflect physical constraints in applications. Such positive systems appear for example in modelling bio-systems, chemical reaction systems, and socio-economic systems, as described in detail in the monograph [10]. A thorough overview of the positive realization problem and related results has recently been given in [3].

It is well known, although maybe surprising, that the constraint of positivity may force the dimension $N$ of realizations to be strictly larger than $n$ (see [2], [5], [15] for different reasons why this phenomenon may occur). Therefore, the minimality problem (i.e. finding the lowest possible value of $N$ ) is essential, and has been dealt with in a number of recent papers (see [6], [8], [12], [7], [16], [14], [13]). The problem is highly non-trivial and a general algorithm of determining the lowest possible value of $N$ is still not known. Several particular cases are settled in the papers mentioned above. Let us mention here that, in contrast with the minimality problem, the existence problem (i.e. determining whether a transfer function $H(z)$ admits a positive realization or not) has already been solved in [1] and [9] (cf. also [11]).

Recent applications of positive system theory include a MOS-based technology for discrete-time filtering (the so-called charge routing networks, see [7]), and the design of fiber optic filters [4]. In fact, the theoretical background in these applications is the following modified version of the positive realization 
problem: decompose an arbitrary transfer function $H(z)$ as the difference $H(z)=H_{1}(z)-H_{2}(z)$ of two transfer functions with positive realizations of dimension $N_{1}, N_{2}$, respectively, and give a priori estimates on the values of $N_{1}$ and $N_{2}$. This problem will be referred to as the positive decomposition problem. For a more detailed description of how the positive realization problem and the positive decomposition problem are related, see the paper [7], and also Section II below.

In this note we are concerned with transfer functions having nonnegative multiple poles. On the one hand, concerning the original positive realization problem, we obtain minimal positive realizations for a class of transfer functions. On the other hand, concerning the positive decomposition problem, we give a generalization of a result of [7]. In fact, the case of multiple poles, in general, is not covered by the method given in [7], and is left open (see the Concluding Remarks of that paper). We attempt to make a step towards the solution by considering the presence of nonnegative multiple poles.

\section{TRAnsfer FunCtions With NONNEGATIVE MUltiple POLES}

In this section we provide an algorithm to find a minimal positive realization for a class of transfer functions with nonnegative multiple poles.

Before stating our results let us make some preliminary remarks. It is well known that a necessary condition for the existence of positive realizations of $H(z)$ is that one of the dominant poles (i.e. poles with maximal modulus) must be nonnegative. The transfer function $H(z)$ is called primitive if it has a unique dominant pole. We will deal only with the case of primitive transfer functions with a simple dominant pole at $\lambda_{0} \geq 0$. We will exclude the trivial case $\lambda_{0}=0$ and assume that $\lambda_{0}>0$.

Throughout the paper we will also use the trivial fact that the impulse response sequence $t_{1}, t_{2}, \ldots$ of a first-order transfer function $T(z)=\frac{c}{z-\mu},(c \in \mathbb{R}, \mu \geq 0)$ is given by $t_{j}=c \mu^{j-1}$.

We will also need the following general result of Hadjicostis (see [12], Theorem 5):

Lemma 1: Let $T(z)$ be a rational transfer function with nonnegative impulse response sequence $t_{1}, t_{2}, \ldots$, i.e. $T(z)=\sum_{j=1}^{\infty} t_{j} z^{-j}$. For an index $m \geq 1$ let $T_{m}(z)$ denote the transfer function corresponding to the truncated sequence $t_{m}, t_{m+1}, \ldots$, i.e. $T_{m}(z)=\sum_{j=1}^{\infty} t_{m+j-1} z^{-j}$. Assume that $T_{m}(z)$ admits a nonnegative realization of dimension $k$. Then $T(z)$ admits a nonnegative realization of dimension $k+m-1$.

The proof of this lemma is a direct construction (see [12] for the details, and Example 1 below for an application).

We can now state our first result concerning transfer functions with nonnegative multiple poles.

Theorem 1: Consider the transfer function

$$
H(z):=\frac{c_{0}}{z-\lambda_{0}}+\frac{c_{1}}{\left(z-\lambda_{1}\right)^{l}},
$$

where $l \geq 1$ is an integer exponent, $0 \leq \lambda_{1}<\lambda_{0}, c_{0}>0$ and $c_{1} \geq-c_{0}\left(\lambda_{0}-\lambda_{1}\right)^{l-1}$. Then $H(z)$ has a minimal positive realization of dimension $l+1$.

Proof. Introduce the new variable $y=z-\lambda_{1}$, and let $G(y):=H(z)$. The function $G$ has poles shifted to the left by $\lambda_{1}$. In particular, 0 is a pole of $G(y)$. In fact, $H(z)=G(y)=\frac{c_{0}}{y-\left(\lambda_{0}-\lambda_{1}\right)}+\frac{c_{1}}{y^{l}}$. 
The idea now is to 'delete' the pole at 0 in the following way. The impulse response sequence $g_{1}, g_{2}, \ldots$ of $G(y)$ (with respect to $y$, of course) is nonnegative (this is a direct consequence of the assumption $c_{1} \geq-c_{0}\left(\lambda_{0}-\lambda_{1}\right)^{l-1}$; namely, for $j \neq l$ we have $g_{j}=c_{0}\left(\lambda_{0}-\lambda_{1}\right)^{j-1}>0$, and $\left.g_{l}=c_{0}\left(\lambda_{0}-\lambda_{1}\right)^{l-1}+c_{1} \geq 0\right)$.

Now simply disregard the terms $g_{1}, \ldots g_{l}$, thus obtaining the sequence $g_{l+1}, g_{l+2}, \ldots$ This impulse response corresponds to the first-order transfer function $G_{l+1}(y):=\frac{\left(\lambda_{0}-\lambda_{1}\right)^{l}}{y-\left(\lambda_{0}-\lambda_{1}\right)}$. The function $G_{l+1}(y)$ has a trivial positive realization in 1 dimension, namely $\mathbf{A}_{\mathbf{1}}=\lambda_{0}-\lambda_{1}, \mathbf{b}_{\mathbf{1}}=1, \mathbf{c}_{\mathbf{1}}=\left(\lambda_{0}-\lambda_{1}\right)^{l}$. We can then apply Lemma 1 to construct a positive realization $\left(\mathbf{A}_{\mathbf{2}}, \mathbf{b}_{\mathbf{2}}, \mathbf{c}_{\mathbf{2}}\right)$ of $G(y)$ in $l+1$ dimension. After this we can define $\mathbf{A}:=\mathbf{A}_{\mathbf{2}}+\lambda_{1} \mathbf{I}, \mathbf{b}=\mathbf{b}_{\mathbf{2}}, \mathbf{c}=\mathbf{c}_{\mathbf{2}}$ and we obtain a positive realization $(\mathbf{A}, \mathbf{b}, \mathbf{c})$ of $H(z)$, also in $l+1$ dimension. To verify this we can simply write

$$
\mathbf{c}^{T}(z \mathbf{I}-\mathbf{A})^{-1} \mathbf{b}=\mathbf{c}_{\mathbf{2}}{ }^{T}\left(\left(z-\lambda_{1}\right) \mathbf{I}-\mathbf{A}_{\mathbf{2}}\right)^{-1} \mathbf{b}_{\mathbf{2}}=\mathbf{c}_{\mathbf{2}}{ }^{T}\left(y \mathbf{I}-\mathbf{A}_{\mathbf{2}}\right)^{-1} \mathbf{b}_{\mathbf{2}}=G(y)=H(z) .
$$

Remark 1. It is clear that this realization is minimal, because the dimension equals the McMillan degree.

Remark 2. It would be very interesting to see whether the sufficient condition on $c_{0}, c_{1}$ above is also necessary or not. It is certainly so in the trivial case $l=1$, but even the simplest non-trivial case $l=2$ seems to be open.

One advantage of the construction of Theorem 1 is that it can also be directly applied in the case when different exponents of the same pole are present.

Corollary 1: Let

$$
H(z)=\frac{c_{0}}{z-\lambda_{0}}+\sum_{j=1}^{l} \frac{c_{j}}{\left(z-\lambda_{1}\right)^{j}},
$$

where $0 \leq \lambda_{1}<\lambda_{0}, c_{0}>0$ and $c_{j} \geq-c_{0}\left(\lambda_{0}-\lambda_{1}\right)^{j-1}$ for all $1 \leq j \leq l$. Then $H(z)$ has a positive realization of dimension $l+1$.

Proof. The method of the proof of Theorem 1 applies directly, as descried in Example 1 below.

Before giving a numerical example, we remark that in positive system theory it is customary to assume (without loss of generality) that the dominant pole $\lambda_{0}=1$, and its residue $c_{0}=1$. What makes this normalization possible is that for any positive constants $a_{1}, a_{2}$ a transfer function $H(z)$ has a positive realization of some dimension $N$ if and only if the function $a_{1} H\left(a_{2} z\right)$ has a positive realization of the same dimension $N$ (see e.g.[1] for the easy proof). We will also use this standard normalization in Example 1 below.

Example 1. Let $H(z)=\frac{1}{z-1}-\frac{0.2}{(z-0.4)^{3}}-\frac{0.3}{z-0.4}$. Introducing $y=z-0.4$ we get $G(y)=\frac{1}{y-0.6}-\frac{0.2}{y^{3}}-\frac{0.3}{y}$. Then $G(y)$ has impulse response sequence $0.7,0.6,0.16, \frac{27}{125}, \ldots, \frac{3^{k-1}}{5^{k-1}} \ldots$ Now we disregard the first three elements of this sequence, and observe that the rest $\frac{27}{125}, \ldots, \frac{3^{k-1}}{5^{k-1}} \ldots$ is the impulse response of the first-order transfer function $G_{4}(y)=\frac{0.216}{y-0.6}$, which has a 1-dimensional positive realization $\mathbf{c}_{\mathbf{1}}=0.216$, $\mathbf{A}_{\mathbf{1}}=0.6$ and $\mathbf{b}_{\mathbf{1}}=1$. We now apply the general method of [12] in order to obtain a positive realization of $G(y)$. Namely, we define (in the notation below we indicated the general construction of [12], although the 'vectors' $\mathbf{b}_{\mathbf{1}}, \mathbf{c}_{\mathbf{1}}$ and the 'matrix' $\mathbf{A}_{\mathbf{1}}$ are in fact scalars in our case): $\mathbf{c}_{\mathbf{2}}{ }^{T}=\left(g_{3}, g_{2}, g_{1}, \mathbf{c}_{\mathbf{1}}{ }^{T}\right)=$ 
$(0.16,0.6,0.7,0.216)$ and $\mathbf{b}_{\mathbf{2}}=\left(0,0,1, \mathbf{0}^{T}\right)^{T}=(0,0,1,0)^{T}$, and

$$
\mathbf{A}_{\mathbf{2}}=\left(\begin{array}{cccc}
0 & 1 & 0 & \mathbf{0}^{T} \\
0 & 0 & 1 & \mathbf{0}^{T} \\
0 & 0 & 0 & \mathbf{0}^{T} \\
\mathbf{b}_{\mathbf{1}} & \mathbf{0} & \mathbf{0} & \mathbf{A}_{\mathbf{1}}
\end{array}\right)=\left(\begin{array}{cccc}
0 & 1 & 0 & 0 \\
0 & 0 & 1 & 0 \\
0 & 0 & 0 & 0 \\
1 & 0 & 0 & 0.6
\end{array}\right)
$$

Finally, the required positive realization $(\mathbf{A}, \mathbf{b}, \mathbf{c})$ of $H(z)$ is achieved by $\mathbf{c}=\mathbf{c}_{\mathbf{2}}, \mathbf{b}=\mathbf{b}_{\mathbf{2}}$, and

$$
\mathbf{A}=\mathbf{A}_{2}+0.4 \mathbf{I}=\left(\begin{array}{cccc}
0.4 & 1 & 0 & 0 \\
0 & 0.4 & 1 & 0 \\
0 & 0 & 0.4 & 0 \\
1 & 0 & 0 & 1
\end{array}\right)
$$

One can have the impression that Theorem 1 and Corollary 1 can only be directly applied to a relatively small class of transfer functions because the conditions are rather restrictive. However, the iteration of the construction above leads us to a more general case. Namely, we can obtain a minimal positive realization of transfer functions with several distinct nonnegative multiple poles. The statement of this result is given in the spirit of [7], keeping in mind subsequent applications to the positive decomposition problem (see Corollary 2 below).

Theorem 2: Let

$$
H(z)=\frac{R}{z-\lambda_{0}}+\sum_{j=1}^{r} \sum_{k=1}^{l_{j}} \frac{c_{j, k}}{\left(z-\lambda_{j}\right)^{k}},
$$

where $R>0,0 \leq \lambda_{j}<\lambda_{0}$ for all $1 \leq j \leq r$ (the poles $\lambda_{j}$ are assumed to be pairwise distinct), and $c_{j, k} \in \mathbb{R}$. If $R$ is sufficiently large, then there exists a minimal positive realization of $H(z)$ of dimension equalling the McMillan degree $n=1+\sum_{j=1}^{r} l_{j}$.

Proof. The idea of the construction in this general case is the iteration of the method described in Theorem 1. This is illustrated by Example 2 below.

The formal proof proceeds by induction with respect to $r$.

For $r=1$ the statement is contained in Corollary 1.

Assume $r>1$, and that the inductive assumption is fulfilled for $r-1$.

We will need the following general observation: if a transfer function $T(z)$ has impulse response sequence $t_{1}, t_{2}, \ldots$ (i.e. $T(z)=\sum_{j=1}^{\infty} t_{j} z^{-j}$ ), then the truncated sequence $t_{2}, t_{3}, \ldots$ is the impulse response of the transfer function $T_{2}(z):=\sum_{j=1}^{\infty} t_{j+1} z^{-j}=z T(z)-t_{1}$. Notice that the non-zero poles of $T_{2}(z)$ (and their orders) are the same as those of $T(z)$. On the other hand, if 0 is a pole of $T(z)$ of order $k \geq 1$ then the order of the pole 0 for $T_{2}(z)$ is $k-1$.

We can assume without loss of generality that $\lambda_{1}<\lambda_{2}<\cdots<\lambda_{r}$. Introduce the new variable $y=z-\lambda_{1}$, and let $G(y)=H(z)$. Assume now that the impulse response sequence $g_{1}, g_{2}, \ldots$ of $G(y)$ (with repect to $y$, of course) is nonnegative. It is clear that this nonnegativity condition will be satisfied if $R>0$ is chosen sufficiently large. We will need the nonnegativity of the sequence $g_{1}, g_{2}, \ldots$ when 
applying Lemma 1 later. Note also that the function $G(y)$ has poles of order $1, l_{r}, l_{r-1}, \ldots, l_{2}, l_{1}$ at locations $\lambda_{0}-\lambda_{1}, \lambda_{r}-\lambda_{1}, \lambda_{r-1}-\lambda_{1}, \ldots, \lambda_{2}-\lambda_{1}, 0$, respectively. We can now proceed by deleting the first $l_{1}$ terms of the impulse response sequence of $G(y)$. By the general observation above we conclude that the truncated sequence $g_{l_{1}+1}, g_{l_{1}+2}, \ldots$ corresponds to some transfer function $G_{l_{1}+1}(y)$ with poles of order $1, l_{r}, \ldots, l_{2}$ at locations $\lambda_{0}-\lambda_{1}, \lambda_{r}-\lambda_{1}, \ldots \lambda_{2}-\lambda_{1}$, respectively. In particular, the pole at 0 has 'disappeared'. In fact, the function $G_{l_{1}+1}(y)$ is of the form

$$
G_{l_{1}+1}(y)=\frac{R^{\prime}}{y-\left(\lambda_{0}-\lambda_{1}\right)}+\sum_{j=2}^{r} \sum_{k=1}^{l_{j}} \frac{d_{j, k}}{\left(y-\left(\lambda_{j}-\lambda_{1}\right)\right)^{k}}
$$

for some $R^{\prime}>0$ and $d_{j, k} \in \mathbb{R}$. Therefore, the inductive hypothesis applies, and a positive realization $\mathbf{A}_{\mathbf{1}}, \mathbf{b}_{\mathbf{1}}, \mathbf{c}_{\mathbf{1}}$ of $G_{l_{1}+1}(y)$ of dimension $1+\sum_{j=2}^{r} l_{j}$ exists. Then, an application of Lemma 1 provides a positive realization $\mathbf{A}_{\mathbf{G}}, \mathbf{b}_{\mathbf{G}}, \mathbf{c}_{\mathbf{G}}$ of $G(y)$ of dimension $1+\sum_{j=1}^{r} l_{j}$. Finally, it is clear that any positive realization $\mathbf{A}_{\mathbf{G}}, \mathbf{b}_{\mathbf{G}}, \mathbf{c}_{\mathbf{G}}$ of $G(y)$ leads to a positive realization $\mathbf{A}_{\mathbf{H}}:=\mathbf{A}_{\mathbf{G}}+\lambda_{1} \mathbf{I}, \mathbf{b}_{\mathbf{H}}:=\mathbf{b}_{\mathbf{G}}, \mathbf{c}_{\mathbf{H}}:=\mathbf{c}_{\mathbf{G}}$ of $H(z)$ of the same dimension.

It is also clear that this construction supplies a minimal positive realization of $H(z)$, because the dimension equals the McMillan degree.

We illustrate the construction described above by the following

Example 2. Let

$$
H(z)=\frac{10}{z-1}-\frac{1}{(z-0.8)^{2}}+\frac{2}{z-0.7}-\frac{0.5}{(z-0.7)^{3}}
$$

Introducing the new variable $y=z-0.7$ we get $G(y):=H(z)=\frac{10}{y-0.3}-\frac{1}{(y-0.1)^{2}}+\frac{2}{y}-\frac{0.5}{y^{3}}$. Then, disregarding the first 3 terms $g_{1}=12, g_{2}=2, g_{3}=0.2$ of the impulse response of $G(y)$ we obtain $G_{4}(y)=\frac{0.27}{y-0.3}+\frac{0.002-0.03 y}{(y-0.1)^{2}}$.

Introducing the new variable $v=y-0.1$ we get $F(v):=G_{4}(y)=\frac{0.27}{v-0.2}-\frac{0.03}{v}-\frac{0.001}{v^{2}}$. Disregarding the first 2 terms $f_{1}=0.24, f_{2}=0.053$ of the impulse response of $F(v)$ we obtain $F_{3}(v)=\frac{0.0108}{v-0.2}$.

The first order transfer function $F_{3}(v)$ has a trivial positive realization, namely $\mathbf{A}_{\mathbf{1}}=0.2, \mathbf{b}_{\mathbf{1}}=1$, $\mathbf{c}_{\mathbf{1}}=0.0108$. Applying Lemma 1 we get a positive realization of $F(v)$, namely

$$
\mathbf{A}_{2}:=\left(\begin{array}{ccc}
0 & 1 & 0 \\
0 & 0 & 0 \\
1 & 0 & 0.2
\end{array}\right)
$$

and $\mathbf{b}_{\mathbf{2}}=(0,1,0)^{T}, \mathbf{c}_{\mathbf{2}}{ }^{T}=(0.053,0.24,0.0108)$. Then, a positive realization of $G_{4}(y)$ is given by

$$
\mathbf{A}_{3}=\mathbf{A}_{2}+0.1 \mathbf{I}=\left(\begin{array}{ccc}
0.1 & 1 & 0 \\
0 & 0.1 & 0 \\
1 & 0 & 0.3
\end{array}\right)
$$

and $\mathbf{b}_{3}=\mathbf{b}_{2}, \mathbf{c}_{3}=\mathbf{c}_{2}$. 
Next we apply Lemma 1 again to obtain a positive realization of $G(y)$, namely

$$
\mathbf{A}_{4}:=\left(\begin{array}{cccccc}
0 & 1 & 0 & 0 & 0 & 0 \\
0 & 0 & 1 & 0 & 0 & 0 \\
0 & 0 & 0 & 0 & 0 & 0 \\
0 & 0 & 0 & 0.1 & 1 & 0 \\
1 & 0 & 0 & 0 & 0.1 & 0 \\
0 & 0 & 0 & 1 & 0 & 0.3
\end{array}\right)
$$

and $\mathbf{b}_{\mathbf{4}}=(0,0,1,0,0,0)^{T}, \mathbf{c}_{\mathbf{4}}{ }^{T}=(0.2,2,12,0.053,0.24,0.0108)$.

Finally, we arrive at a minimal positive realization of $H(z)$, namely

$$
\mathbf{A}_{5}=\mathbf{A}_{4}+0.7 \mathbf{I}:=\left(\begin{array}{cccccc}
0.7 & 1 & 0 & 0 & 0 & 0 \\
0 & 0.7 & 1 & 0 & 0 & 0 \\
0 & 0 & 0.7 & 0 & 0 & 0 \\
0 & 0 & 0 & 0.8 & 1 & 0 \\
1 & 0 & 0 & 0 & 0.8 & 0 \\
0 & 0 & 0 & 1 & 0 & 1
\end{array}\right)
$$

and $\mathbf{b}_{5}=\mathbf{b}_{4}, \mathbf{c}_{5}=\mathbf{c}_{4}$.

Although the result of Theorem 2 is rather vague with respect to the original minimality problem of positive realizations (i.e. it does not provide an explicit estimate on how large the value of $R$ must be compared to the coefficients $c_{j, k}$ ), it can be appreciated better in connection with the positive decomposition problem (which, in turn, may even be more important in applications, as described in [7] and [4]). Namely, the case of transfer functions with multiple poles was left as an open problem in the Concluding Remarks of [7], and we can now make a small step towards the solution. The combination of the result of Theorem 8 in [7] and our Theorem 2 above gives the following

Corollary 2: Let $H(z)$ be a strictly proper asymptotically stable rational transfer function (i.e. its poles lie in the interior of the unit disk), with arbitrary simple (possibly complex) poles, and nonnegative poles of possibly higher order. Let $N_{1}$ denote the sum of the orders of the nonnegative poles, and $N_{2}$ the number of negative real poles. Let $\mathcal{P}_{j}(j \geq 3)$ denote the set of points in the complex plane that lie in the interior of the regular polygon with $j$ edges having one vertex in point 1 and center at 0 . $\mathcal{P}_{j}$ can formally be defined in polar coordinates as in [7]:

$$
\mathcal{P}_{j}:=\left\{(\rho, \theta): \rho \cos \left[\frac{(2 k+1) \pi}{j}-\theta\right]<\cos \frac{\pi}{j} \quad \text { for all } k=0,1, \ldots, j-1\right\} .
$$

Let $N_{3}$ denote the number of pairs of complex conjugate poles of $H(z)$ belonging to the region $\mathcal{P}_{3}$. Further, let $N_{j}(j \geq 4)$ denote the number of pairs of complex conjugate poles of $H(z)$ belonging to the region

$$
\mathcal{P}_{j} \backslash \cup_{m=3}^{j-1} \mathcal{P}_{m}
$$


Then $H(z)$ can be realized as the difference $H(z)=H_{1}(z)-H_{2}(z)$ of an $N$ dimensional positive system $H_{1}$ and a 1-dimensional positive system $H_{2}$ with

$$
N=1+N_{1}+\sum_{j \geq 2} j N_{j}
$$

Proof. This result is a direct consequence of Theorem 8 in [7] and our Theorem 2 above. For convenience we repeat the major points of the argument.

Write $H(z)$ in the form $H(z)=H_{1}(z)-H_{2}(z)$, where $H_{2}(z):=\frac{R}{z-1}$ with $R>0$ sufficiently large, and $H_{1}(z)=\frac{R}{z-1}+H(z)$. Then $H_{2}(z)$ is a one-dimensional positive system, therefore it is enough to construct a positive realization of $H_{1}(z)$ of the prescribed dimension $N$.

Consider the partial fraction decomposition of $H(z)$. Let $H_{+}(z)$ denote the sum of partial fractions corresponding to nonnegative (possibly multiple) poles. In view of Theorem 2 above, we can find a sufficiently large positive number $R_{1}$ such that the function $\frac{R_{1}}{z-1}+H_{+}(z)$ has a positive realization of dimension $1+N_{1}$. The sum of the remaining partial fractions $H_{-}(z)$ contains simple poles only (in fact, only negative real poles and pairs of complex conjugate poles remain). Therefore, the result of [7], Theorem 8 can directly be applied. Namely, for a sufficiently large positive number $R_{2}$ the function $\frac{R_{2}}{z-1}+H_{-}(z)$ has a positive realization of dimension $\sum_{j \geq 2} j N_{j}$. We can now choose $R=R_{1}+R_{2}$ and the parallel connection of the positive realization of $\frac{R_{1}}{z-1}+H_{+}(z)$ and $\frac{R_{2}}{z-1}+H_{-}(z)$ gives the desired result.

Note that in Corollary 2 we cannot claim minimality of the obtained realization (cf. [7]). However, the main point, as explained in more detail in [7], is that we have a reasonably good a priori upper estimate on the dimension of the realization.

It is also clear that the case of negative real or pairs of complex conjugate multiple poles falls outside the scope of our considerations and remains open.

\section{CONCLUSION}

In this brief we provided a construction to obtain a minimal and positive realization for a class of transfer functions with nonnegative multiple poles. The results also enabled us to generalize an existing result in positive system theory concerning the positive decomposition problem.

\section{REFERENCES}

[1] B.D.O. Anderson, M. Deistler, L. Farina, and L. Benvenuti, "Nonnegative realization of a linear system with nonnegative impulse response," IEEE Trans. Circuits Syst. I, vol. 43, no.2, pp 134-142, Feb. 1996.

[2] B.D.O. Anderson, New developments in the theory of positive systems in: Byrnes, Datta, Gilliam, Martin, (Eds.) Systems and Control in the Twenty-First Century, Birkhäuser, Boston 1997.

[3] L. Benvenuti and L. Farina, "A tutorial on the positive realization problem", IEEE Trans. Automat. Control vol. 49, no. 5, pp. 651-664, 2004.

[4] L. Benvenuti and L. Farina, "The design of fiber-optic filters", Journal of Lightwave Technology, vol. 19, pp. 1366-1375, 2001.

[5] L. Benvenuti, L. Farina, "An example of how positivity may force realizations of 'large' dimensions," Syst. Contr. Lett., vol. 36, pp. 261-266, 1999. 
[6] L. Benvenuti and L. Farina, A note on minimality of positive realizations, IEEE Trans. Circuits Syst. I, vol. 45, pp. 676-677, June 1998.

[7] L. Benvenuti, L. Farina, and B.D.O. Anderson, "Filtering through a combination of positive filters," IEEE Trans. Circuits Syst. I, vol. 46, pp. 1431-1440, Dec. 1999.

[8] L. Benvenuti, L. Farina, B. D. O. Anderson and F. De Bruyne, Minimal positive realizations of transfer functions with positive real poles, IEEE Trans. Circuits Syst. I, vol. 47, pp. 1370-1377, Sept. 2000.

[9] L. Farina, "On the existence of a positive realization," Syst. Contr. Lett., vol. 28, no.4, pp 219-226, 1996.

[10] L. Farina and S. Rinaldi, Positive linear systems: Theory and applications. New York: Wiley, 2000.

[11] K.-H. Frster and B. Nagy, "Nonnegative realizations of matrix transfer functions", Linear Algebra Appl. vol. 311, no. 1-3, pp. 107-129, 2000.

[12] C. Hadjicostis, "Bounds on the size of minimal nonnegative realizations for discrete-time LTI systems," Syst. Contr. Lett., vol. 37, pp 39-43, 1999.

[13] A. Halmschlager and M. Matolcsi, "Minimal positive realizations for a class of transfer functions", IEEE Trans. Circuits Syst. II, to appear.

[14] J. M. van den Hof, Realizations of positive linear systems, Linear Algebra and its Applications, vol. 256, pp. 287-308, 1997.

[15] B. Nagy, M. Matolcsi, "A lowerbound on the dimension of positive realizations," IEEE Trans. Circuits Syst. I, vol. 50, pp. 782-784, June, 2003.

[16] G. Picci, J. M. van den Hof and J. H. van Schuppen, "Primes in several classes of the positive matrices," Linear Algebra and its Applications, vol. 277/13, pp. 149-185, 1998. 